\title{
Optical Fibre Sensors based on Multi-Mode fibres and MIMO signal processing: An experimental Approach
}

\author{
Andreas Ahrens ${ }^{a}$, Andre Sandmann ${ }^{a}$, Kort Bremer ${ }^{b}$, Bernhard Roth $^{b}$, Steffen Lochmann ${ }^{a}$ \\ ${ }^{a}$ Wismar University, Philipp-Müller Strasse 14, 23966 Wismar, Germany; \\ ${ }^{b}$ Leibniz University Hannover, Nienburger Strasse 17, 30167 Hannover, Germany
}

\begin{abstract}
In this paper multiple-input multiple-output (MIMO) signal processing is investigated for fibre optic sensor applications. A $(2 \times 2)$ MIMO implementation is realized by using lower-order and higher-order mode groups of a graded-index (GI) multi-mode fibre (MMF) as separate transmission channels. A micro-bending pressure sensor changes these separate transmission characteristics and introduces additional crosstalk. By observing the weight-factors of the MIMO system the amount of load applied was determined. Experiments verified a good correlation between the change of the MIMO weight coefficients and the load applied to the sensor and thus verified that MIMO signal processing can beneficially be used for fibre optic sensor applications.
\end{abstract}

Keywords: Multiple-Input Multiple-Output System, Singular-Value Decomposition, Optical Fibre Sensors.

\section{INTRODUCTION}

Fibre optic sensors have the inherent advantage of being immune to Electromagnetic Interference (EMI), small in size, robust, resistance to corrosion, electrically passive and easy to multiplex. To date different concepts of fibre optic sensors have been reported based on e.g. Fibre Bragg Gratings (FBGs), intensity modulated and interferometric sensors as well as on Brillouin, Raman and Rayleigh scattering. Due to the advantages of fibre optic sensors, they have been already applied successfully to measure the structural health of structures such as bridges, dams, mines or advanced composite materials in aircrafts. Furthermore, they have been used to monitor the load of power transmission lines and temperature as well as pressure in oil and gas wells. ${ }^{1}$ Though fibre optic sensors have reached a high state of maturity and their fields of application are wide spread more efficient signal processing procedures are still important. In the field of optical communications spatial division multiplexing (SDM) is currently investigated in order to overcome the capacity limit of common single-mode fibres (SMF). One approach of SDM utilizes individual transversal modes of multi-mode fibres (MMF) for data transmission. However, due to external perturbations such as bending and elongation of the optical fibre along the optical MMF link and fibre imperfections cross-talk occur between the transversal modes and thus multiple-input-multipleoutput (MIMO) signal processing has to be applied at the output in order to recover each orthogonal signal at the input. Placing a perturbation device like a micro-bending sensor in this path not only higher transmission losses but also strong mode coupling occur depending on the measurand. In this paper MIMO signal processing is experimentally explored for fibre optical sensor applications. The experiments are based on a $(2 \times 2)$ MIMO implementation which has been realized by using lower-order and higher-order mode groups of a graded-index (GI)-MMF as separate transmission channels. Mode coupling and therefor crosstalk was obtained between the transmission channels by introducing a defined and repeatable perturbation, i.e. by applying a micro-bender and different forces at a certain position along the optical fibre MIMO link. By observing the weight-function of the MIMO system, the amount of perturbation $i$. e. the amount of force applied was determined.

\section{OPTICAL MIMO}

The principle of optical MIMO is based on the activation of different transversal modes or mode groups of MMF's as individual data transmission channels and thus to realize parallel data transmission over a single MM fibre link. An implementation of a $(2 \times 2)$ optical MIMO is based on the utilization of low-order and high-order mode groups as individual transmission channels. These different mode-groups travel together in a MMF and can be separated at the receiver by using spatial mode filters. A schematic of the spatial mode filters is illustrated in Fig.1.

24th International Conference on Optical Fibre Sensors, edited by Hypolito José Kalinowski, José Luís Fabris, Wojtek J. Bock, Proc. of SPIE Vol. 9634, 96345W · C 2015 SPIE CCC code: $0277-786 \mathrm{X} / 15 / \$ 18 \cdot$ doi: $10.1117 / 12.2195114$

Proc. of SPIE Vol. 9634 96345W-1 


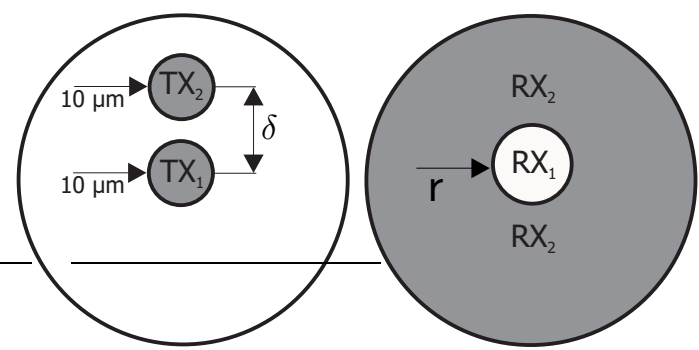

Figure 1. Forming the optical MIMO channel (left: light launch positions at the transmitter side with a given eccentricity $\delta$, right: spatial configuration at the receiver side as a function of the mask radius $r$ )

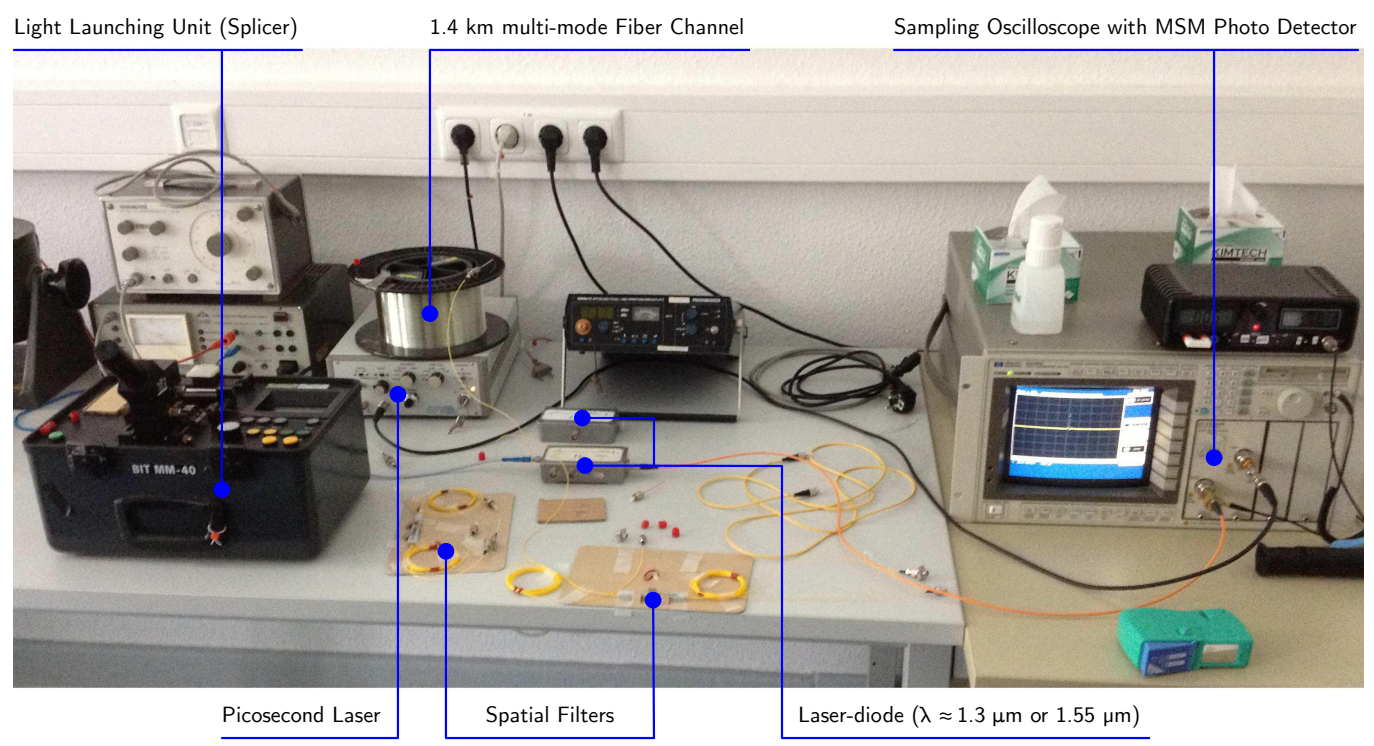

Figure 2. Measurement setup for determining the MIMO specific impulse responses.

The spatial mode filters have been fabricated by initially coating the end-face of a grated-index MMF with gold and then partly removing the coating again by using Focused Ion Beam (FIB) milling. The resulting spatial mode filter only allows light of a certain spatial distribution to reach the detector. All other light is reflected back into the fibre. Moreover, the excitation of the different mode-groups can be done through various methods. Besides using Spatial Light Modulators (SLM), Long-Period Gratings (LPG) or Photonic Crystals, the excitation can simply be carried out by a centric or an eccentric splice between a single-mode fibre (SMF) and a MMF. The measurement setup depicted in Fig. 2 shows the testbed with the utilized devices for measuring the system properties of the optical MIMO channel in form of its specific impulse responses needed for modelling the MIMO data transmission.

\section{SYSTEM MODEL}

The block diagram of the transmission model is shown in Fig. 3. The block-oriented system for frequency selective channels is modelled by: $\mathbf{u}=\mathbf{H} \cdot \mathbf{c}+\mathbf{w}$, where the transmitted signal vector $\mathbf{c}$ is mapped by the channel matrix $\mathbf{H}$ onto the received vector $\mathbf{u}$. Finally, the vector of the additive, white Gaussian noise (AWGN) is defined by w. ${ }^{2}$ Details on the transmission model, which has been determined by channel measurements, are given in. ${ }^{2}$ In MIMO communication, singular-value decomposition (SVD) has been established as an efficient concept to compensate the interferences between the different data streams transmitted over a dispersive channel: SVD is able to transfer the whole system into independent, non-interfering layers exhibiting unequal gains per layer as highlighted in Fig. 4. By applying the MIMO concept to optical fibre sensors based on intensity measurements 


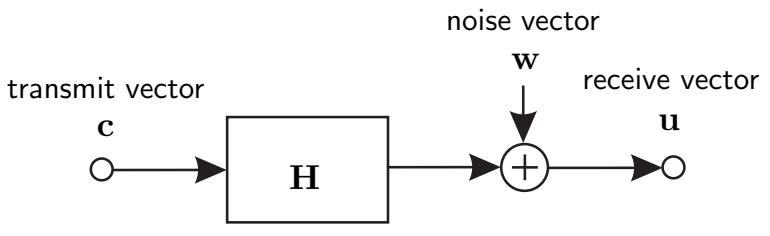

Figure 3. Transmission system model

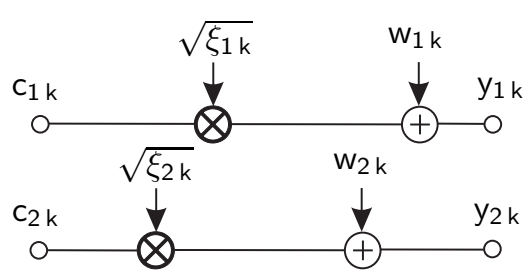

Figure 4. SVD-based layer-specific transmission model

several advantages can be achieved. In standard measurement conditions with full mode excitation mainly the higher order modes disappear due-to perturbations leading to relatively small, i. e. less sensitive power changes in comparison to the main power carried by the other modes. Thus, exciting only individual modes or mode groups results in a much higher sensor sensitivity, e. g. three to six times as shown in. ${ }^{3}$ However, the measuring range of the sensor is also reduced since no optical power is left after the disappearance of the information carrying order mode. Now, MIMO processing has the potential capacity of making use of all the individually launched modes which are transformed from lower to higher order modes in correlation with the disturbance. Thus both a higher sensitivity and a wider measuring range are achieved.

\section{EXPERIMENTAL SET-UP}

The experimental set-up shown in Fig. 1 was established in order to investigate whether MIMO signal processing in combination with mode-group multiplexing of a GI-MMF can be applied for optical fibre sensor applications. As illustrated in Fig. the experimental set-up consists of a laser-diode (LD) operating at a wavelength of $1327 \mathrm{~nm}, 1 \mathrm{~m}$ of single-mode fibre (SMF) followed by a microbender (MB) using a $2 \mathrm{~m}$ GI-MMF, a coil of GI-MMF with a length of $1400 \mathrm{~m}$, a 40-GHz detector (Det), a 50-GHz digital sampling oscilloscope (DSO), a pulse generator (PS) (Picosecond Diode Laser System), a position controller (PC) as well as a spatial mode (MF) filter in front of the detector. The PC was realised by using the positioning unit of a fusion splicer (BIT

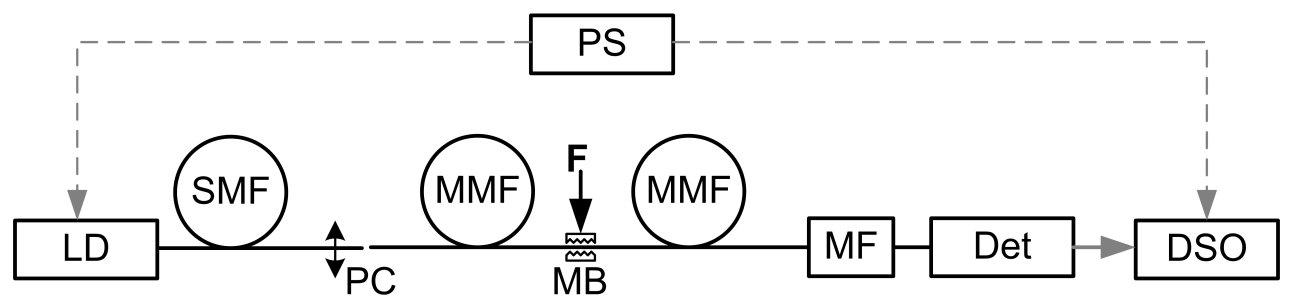

Figure 5. Experimental set-up in order to demonstrate the MIMO can be applied for optical fibre applications as well

MM-40) and was applied in order to excite different mode-groups by changing the off-set position (usually to 10 $\mu \mathrm{m}$ ) of the SMF relative to the centre of the GI-MMF. The MF only allows light of a certain spatial distribution, i.e. light only from the lower-order or higher-order mode-groups, to reach the detector. The MF have been fabricated by initially coating the end-face of a grated-index MMF with gold and then partly removing the coating again by using Focused Ion Beam (FIB) milling. The positons of the SMF relative to the centre of the MMF core to excite the lower respective higher-order mode groups and the layouts of the two different MF are shown in Fig.1. The MB has been realized by two opposite metal plates with corrugated surfaces. Both metal plates are separated by the GI-MMF and the periodic perturbations of the two metal plates are facing each other. Therefore, depending on the applied force, micro-bends are introduced into the GI-MMF and thus light is coupled between the mode-groups of the GI-MMF. For the experiments two MB units with five and ten teeth have been utilized, respectively. The pitch of the periodic perturbations is $1 \mathrm{~mm}$ which ensures optimum mode coupling. The force applied to the MB was adjusted by using high-precision weights.

\section{RESULTS}

The numerical analysis targets at the correlation of the layer-specific weighting factors with the measurand. Each MB unit has been loaded with different weights and the impulse responses have been measured subsequently. ${ }^{2}$ 
In Fig. 6 and 7 the measured impulse responses are illustrated for a MB unit with ten teeth and 0 gram and 400 gram load, respectively. As illustrated in both figures the peak heights which represent the power carried by individual principal modes are changing due to the applied load. Table 1 illustrates the dependence of the MIMO layer-specific weighting factors to the different loads for the two MB units. Thus a high correlation between an
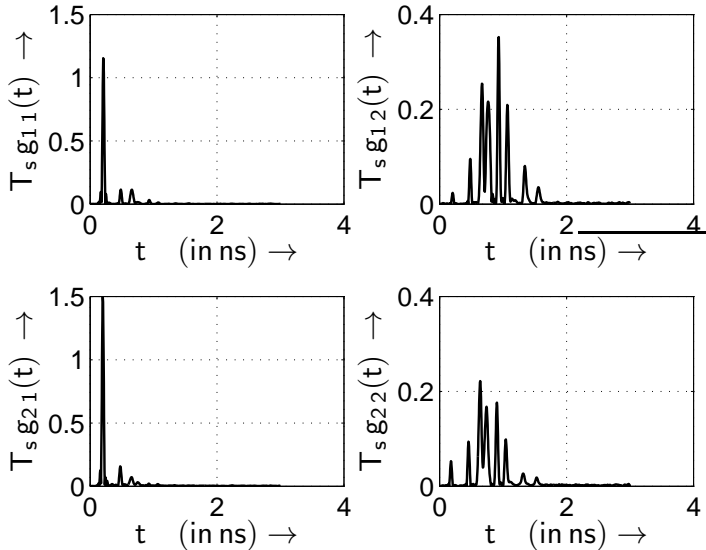

Figure 6. Measured electrical MIMO impulse responses with respect to the pulse frequency $f_{\mathrm{T}}=1 / T_{\mathrm{s}}=5,12$ $\mathrm{GHz}$ at $1325 \mathrm{~nm}$ operating wavelength
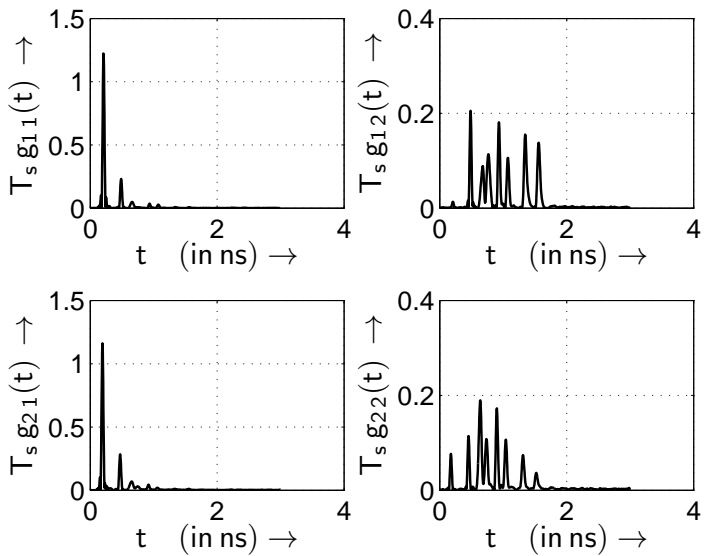

Figure 7. Measured electrical MIMO impulse responses with respect to the pulse frequency $f_{\mathrm{T}}=1 / T_{\mathrm{s}}=5,12$ $\mathrm{GHz}$ at $1325 \mathrm{~nm}$ operating wavelength

optical fibre sensor measurand and MIMO processed data has been confirmed to our knowledge for the first time. The results in Table 1 also reveal a high sensitivity of the five teeth MB unit. The stronger response of this unit can be explained by the higher pressure applied to the GI-MMF. As the surface area of the five teeth MB unit is smaller a higher local pressure is obtained for similar loads.

Table 1. Sensor dependent changes in the layer-specific weighting factors

\begin{tabular}{ccccccccccc}
\hline sensor/weight & 0 & 100 & 200 & 300 & 400 & 500 & 600 & 700 & 800 & 900 \\
\hline 5 teeth & 0,61 & 0,51 & 0,48 & 0,32 & 0,13 & - & - & - & - & - \\
10 teeth & 0,55 & 0,54 & 0,53 & 0,50 & 0,50 & 0,49 & 0,47 & 0,43 & 0,38 & 0,26 \\
\hline
\end{tabular}

\section{CONCLUSIONS}

In this paper MIMO signal processing has been successful investigated experimentally for fibre optic sensor applications. A $(2 \times 2)$ fibre optical MIMO configuration has been realized by using lower-order and higher-order mode groups of a (GI)-MMF. Mode coupling producing crosstalk between the MIMO specific layers has been introduced by applying a MB sensor unit. The crosstalk was controlled by changing the number of teeth of the MB and applying different weights. A high correlation between an optical fibre sensor measurand and MIMO processed data has been confirmed to our knowledge for the first time. The sensitivity to the measurand can be adjusted by the number of teeth of the respective MB sensor unit.

\section{REFERENCES}

[1] Leung, C. K. Y., "Optical Fiber Sensors for Civil Engineering Applications," Materials and Structures 48(4), 871-906 (2015).

[2] Pankow, J., Aust, S., Lochmann, S., and Ahrens, A., "Modulation-Mode Assignment in SVD-assisted Optical MIMO Multimode Fiber Links," in [15th International Conference on Optical Network Design and Modeling (ONDM)], (8.-10. February 2011).

[3] Donlagic, D., "Microbend sensor structure for use in distributed and quasidistributed Sensor Systems based on selective launching and filtering of the Modes in Graded Index Multimode Fiber," Journal of Lightwave Technology 17(10), 1856-1868 (1999). 